\title{
A SOLUTION OF A PROBLEM OF STEENROD FOR CYCLIC GROUPS OF PRIME ORDER
}

\author{
JAMES E. ARNOLD, JR.
}

\begin{abstract}
Given a $Z[G]$ module $A$, we will say a simply connected $\mathrm{CW}$ complex $X$ is of type $(A, n)$ if $X$ admits a cellular $G$ action, and $H_{i}(X)=0$, $i \neq n, H_{n}(X) \simeq A$ as $Z[G]$ modules.

In [5], R. Swan considers the problem posed by Steenrod of whether or not there are finite complexes of type $(A, n)$ for all finitely generated $A$ and finite $G$. Using an invariant defined in terms of $G_{0}(Z[G])$, solutions were obtained for $A=Z_{p}$ (p-prime) and $G \subseteq$ Aut $\left(Z_{p}\right)$. The question of infinite complexes of type $(A, n)$ was left open. In this paper we obtain the following complete solution for $Z\left[Z_{p}\right]$ modules:

There are complexes of type $(A, n)(n \geqslant 3)$, and there are finite complexes of type $(A, n)$ if and only if the invariant which corresponds to Swan's invariant for these modules vanishes.
\end{abstract}

1. Definitions and known results on the problem of Steenrod. If $X$ is a $\mathrm{CW}$ complex, and $G$ a discrete group operating effectively and cellularly on $X$, then we will say $X$ is a $G$-complex. The cellular chain complex of $X$ (denoted $\left.C_{*}(X)\right)$ will then be a $Z[G]$ chain complex, and the homology of $X$ will consist of $Z[G]$ modules.

As a $Z[G]$ module, $C_{n}(X)=H_{n}\left(X^{n}, X^{n-1}\right)$ is a direct sum of the modules determined by the orbits of $n$-cells. Given an $n$-cell $e_{\alpha}^{n}$, let $H_{\alpha}$ denote the subgroup of $G$ fixing $e_{\alpha}^{n}$ (not necessarily pointwise), and define $\varepsilon: H_{\alpha} \rightarrow Z_{2}$ $=\{ \pm 1\}$ by $\varepsilon(h)=$ degree $h:\left(e_{\alpha}^{n}, \partial e_{\alpha}^{n}\right) \rightarrow\left(e_{\alpha}^{n}, \partial e_{\alpha}^{n}\right)$. As in [4] we let $Z_{\varepsilon}$ denote the integers with $Z\left[H_{\alpha}\right]$ module structure defined by $h \cdot n=\varepsilon(h) \cdot n$. The submodule of $C_{n}(X)$ determined by the orbit of $e_{\alpha}^{n}$ is then $Z[G] \otimes_{Z\left[H_{\alpha}\right]} Z_{\varepsilon}$, and $C_{n}(X)$ is a direct sum of such modules. As in [5], we will refer to direct sums of modules of the form $Z[G] \otimes_{Z[H]} Z_{\varepsilon}$ as signed permutation modules.

Given a finite group $G$, we let $G_{0}(Z[G])$ denote the Grothendieck group of finitely generated left $Z[G]$ modules, and $S \subset G_{0}(Z[G])$, the subgroup generated by the signed permutation modules defined above.

Definition (1.1). Let $A$ be a finitely generated $Z[G]$ module. The Swan invariant of $A$, denoted $\mathrm{SW}(A)$, is the class of $A$ in $G_{0}(Z[G]) / S$.

Definition (1.2). Given a $Z[G]$ module $A$, a simply connected $G$-complex $X$ is of type $(A, n)$ if $\tilde{H}_{i}(X)=0, i \neq n, H_{n}(X) \simeq A$ as $Z[G]$ modules.

Received by the editors July 29, 1975 and, in revised form, January 13, 1976.

AMS (MOS) subject classifications (1970). Primary 55C35, 57F99.

Key words and phrases. Cellular group actions, $Z\left[Z_{p}\right]$ modules, Swan's invariant, Grothendieck group.

() American Mathematical Society 1977 
In [5], R. Swan considers the problem posed by Steenrod of whether or not there are finite complexes of type $(A, n)$ for all finitely generated $A$ and finite $G$. Swan defines the invariant $\operatorname{Sw}(A)$, and notes that if there is a finite complex of type $(A, n)$, then $\mathrm{Sw}(A)=0$. This follows from the Euler characteristic formula in $G_{0}(Z[G]),[Z]+(-1)^{n}[A]=\sum(-1)^{i}\left[C_{i}(X)\right]$, and the observation that $[Z]$ and $\left[C_{i}(X)\right]$ are in $S$. Swan obtains solutions to Steenrod's problem in the case that $A=Z_{p}$ a cyclic group of prime order $p$, and $G \subseteq$ Aut $\left(Z_{p}\right)$. In particular, he shows that when $p=47$ and $G \subset$ Aut $\left(Z_{p}\right)$ has order 23, $\mathrm{Sw}(A) \neq 0$, and thus there is no finite complex of type $(A, n)$. The question of infinite complexes of type $(A, n)$ was left open.

Our main theorem is the following complete solution for $Z\left[Z_{p}\right]$ modules:

THEOREM. Let $A$ be a finitely generated $Z\left[Z_{p}\right]$ module, $Z_{p}$ the cyclic group of prime order $p$. Then there are $Z_{p}$-complexes of type $(A, n), n \geqslant 3$, and there is a finite complex of type $(A, n)$ if and only if $\mathrm{Sw}(A)=0$.

2. Results true for all finite groups. In this section we prove several general results on $G$-complexes which will allow some simplification in the type of modules considered when constructing spaces of type $(A, n)$.

LeMma (2.1). Let $X$ and $Y$ be G-complexes where

(a) $X=\bigvee_{\alpha \in A} S_{\alpha}^{n}$ with $G$ permuting the $n$-spheres $S_{\alpha}^{n}$ freely and fixing the base point $x_{0}$; and

(b) $Y$ is $n-1$ connected with a point $y_{0}$ fixed by $G$.

Then any $Z[G]$ homomorphism $h: H_{n}(X) \rightarrow H_{n}(Y)$ is realized by a G-equivariant map $f: X \rightarrow Y$.

Proof. From the hypothesis of the lemma, $H_{n}(X)=\pi_{n}\left(X, x_{0}\right)$ and $H_{n}(Y)$ $\simeq \pi_{n}\left(Y, y_{0}\right)$ as $Z[G]$ modules, and therefore we need only realize the induced homomorphism $\bar{h}: \pi_{n}\left(X, x_{0}\right) \rightarrow \pi_{n}\left(Y, y_{0}\right)$. Choose $B \subset A$ so that the collection $\left\{S_{\beta}^{n}\right\}_{\beta \in B}$ has exactly one sphere from each orbit of $n$-spheres, and let $\left\{\bar{e}_{\beta}\right\}_{\beta \in B}$ be the corresponding classes in $\pi_{n}\left(X, x_{0}\right)$. Let $f\left(x_{0}\right)=y_{0}$, and choose $f$ : $\left(S_{\beta}^{n}, x_{0}\right) \rightarrow\left(Y, y_{0}\right)$ to represent $\bar{h}\left(\bar{e}_{\beta}^{n}\right) \in \pi_{n}\left(Y, y_{0}\right) . f$ is defined on the rest of $X$ by requiring that $f(g(x))=g f(x)$ for all $g \in G$.

REMARK (2.2.) If $y_{0}$ is a 0 -cell of $Y$, then we take $f$ to be cellular by choosing cellular representatives for $\bar{h}\left(\bar{e}_{\beta}\right) \in \pi_{n}\left(Y, y_{0}\right)$.

Lemma (2.3). Let $X$ and $Y$ be G-complexes where

(a) $\operatorname{dim}(X)=n$ and $G$ permutes the $n$-cells of $X$ freely; and

(b) $Y$ is $n-1$ connected, $H_{n}(Y)$ is $Z[G]$ free, and $G$ fixes some point of $Y$.

Then any $Z[G]$ homomorphism $h: H_{n}(X) \rightarrow H_{n}(Y)$ is realized by $a G$ equivariant map $f: X \rightarrow Y$.

Proof. Let $X^{\prime}=X / X^{n-1}$ and note that $X^{\prime}$ and $Y$ satisfy (a) and (b) of Lemma (2.1) respectively. If there is a homomorphism $h^{\prime}: H_{n}\left(X^{\prime}\right) \rightarrow H_{n}(Y)$ so 
that (2.4) commutes,

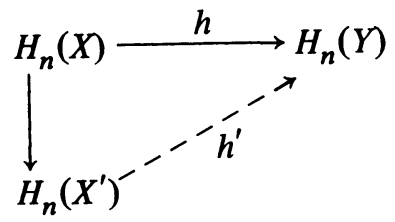

then we can realize $h^{\prime}$ by $f^{\prime}$ and the composite $X \rightarrow X^{\prime} \stackrel{f^{\prime}}{\rightarrow} Y$ will realize $h$.

To find $h^{\prime}$, note that $H_{n}(X)$ is a summand (over $Z$ ) of $H_{n}\left(X^{\prime}\right)$ $=C_{n}(X)$ since $0 \rightarrow H_{n}(X) \rightarrow C_{n}(X) \rightarrow B_{n-1}(X) \rightarrow 0$ is exact, and $B_{n-1}(X)$ (the $n-1$ boundaries) is $Z$-free. Therefore $h$ extends to s $Z$-homomorphism $\bar{h}: H_{n}\left(X^{\prime}\right) \rightarrow H_{n}(Y)$. Now since $H_{n}(Y)$ is $Z[G]$ free, it is weakly injective and we let $h^{\prime}=N(\rho \bar{h})$ where $N$ and $\rho$ are as in [1, Chapter XII]. $h^{\prime}$ satisfies (2.4) and thus we have Lemma (2.3).

REMARK (2.5). Note that if the fixed point of $Y$ is a 0 -cell, we can choose $f$ to be cellular by (2.2).

We make the following definition for applications of Lemma (2.3):

Definition (2.6). $X$ is tractable of type $(A, n)$ if $X$ is an $n$-dimensional $G$ complex of type $(A, n)$, so that $G$ permutes the $n$-cells of $X$ freely and fixes a 0 -cell.

Now suppose $0 \rightarrow B \rightarrow F \rightarrow A \rightarrow 0$ is an exact sequence of $Z[G]$ modules where $F$ is free. Then if $X$ is tractable of type $(B, n)$, we can choose $Y=\vee_{\alpha \in I} S_{\alpha}^{n}$ of type $(F, n)$ and realize $h: H_{n}(X) \rightarrow H_{n}(Y)$ by an equivariant cellular map $f: X \rightarrow Y$. The mapping cone of $f$ will then be a $G$-complex of type $(A, n)$, and has dimension $n+1$.

Given $A$ finitely generated, we can choose an exact sequence $0 \rightarrow B \rightarrow F$ $\rightarrow A \rightarrow 0$ so that $F$ is finitely generated and free. $B$ will then be torsion free (as an abelian group), and $\mathrm{Sw}(B)=-\mathrm{Sw}(A)$. Consequently, the main theorem will be established for a group $G$ whenever we can find tractable realizations for the torsion free modules $B$, and finite tractable realizations for those with $\mathrm{Sw}(B)=0$.

The following observations serve to reduce the problem further:

(2.7) Let $0 \rightarrow B \rightarrow F \stackrel{g}{\rightarrow} A \rightarrow 0$ be exact, and $F$ (finitely generated) free. Then if $(A, n)$ has a (finite) tractable realization, so does $(B, n+1)$.

Proof. Choose $X=\bigvee_{\alpha \in I} S_{\alpha}^{n}$ of type $(F, n)$, and $Y$ (finite) tractable of type $(A, n)$. By Lemma (2.1) there is an equivariant cellular map $f: X \rightarrow Y$ realizing $g$, and the mapping cone of $f$ is tractable of type $(B, n+1)$.

(2.8) If $(A, n)$ has a (finite) tractable realization, so does $(A, n+1)$.

Proof. If $X$ is (finite) tractable of type $n$, let $\sum X$ denote the unreduced suspension of $X$ and define $G \times \sum X \rightarrow \sum X$ by suspension. $\sum X$ is then (finite) tractable of type $(A, n+1)$.

(2.9) If $(A, n)$ and $(B, n)$ have (finite) tractable realizations, then do does $(A \oplus B, n)$. 
Proof. Let $X$ be (finite) tractable of type $(A, n)$, and $Y$ (finite) tractable of type $(B, n)$. Then $X \vee Y$ is (finite) tractable of type $(A \oplus B, n)$ where we take the fixed 0 -cells as wedge point.

3. Properties of $Z\left[Z_{p}\right]$ modules and proof of the main theorem. Let $Z_{p}$ denote the cyclic group of prime order $p$ with generator $t$. If we let $\zeta_{p}=e^{2 \pi i / p}$, and $N$ $=1+t+\cdots+t^{p-1} \in Z\left[Z_{p}\right]$, then $Z\left[Z_{p}\right] /(N) \simeq Z\left[\zeta_{p}\right]$, the ring of $p$ cyclotomic integers. We let $C\left(Z\left[\zeta_{p}\right]\right)$ denote the ideal class group of $Z\left[\zeta_{p}\right]$, i.e. the equivalence classes of nonzero ideals of $Z\left[\zeta_{p}\right]$ under the relation $\mathbb{Q} \sim \mathscr{B}$ $\Leftrightarrow x \cdot Q=y \cdot \mathscr{B}$ for some $0 \neq x, y \in Z\left[\zeta_{p}\right] . C\left(\left[\zeta_{p}\right]\right)$ is a finite abelian group under multiplication, and the class of the principal ideals is the identity.

Using the natural homomorphism, any $Z\left[\zeta_{p}\right]$ module is a $Z\left[Z_{p}\right]$ module. Another way of forming $Z\left[Z_{p}\right]$ modules is the following construction of Reiner:

If $0 \neq \mathbb{Q}$ is an ideal in $Z\left[\zeta_{p}\right]$, then $\mathbb{Q} /\left(\zeta_{p}-1\right) \mathbb{Q} \simeq Z_{p}$. Let $\omega \in \mathbb{Q}, \omega$ $\notin\left(\zeta_{p}-1\right) \mathcal{Q}$, and define $\mathbb{Q}_{\omega}=\mathscr{Q} \oplus Z$ (as abelian groups) and $t \cdot(x, y)$ $=\left(\zeta_{p} x+y \omega, y\right)$. The resulting module $\mathbb{Q}_{\omega}$ is independent of the choice of $\omega$, and there is an exact sequence $0 \rightarrow \mathbb{Q} \rightarrow \mathbb{Q}_{\omega} \rightarrow Z \rightarrow 0$ where $Z$ is the trivial $Z\left[Z_{p}\right]$ module (see [2]). $H^{*}\left(Z_{p} ; \mathbb{Q}_{\omega}\right)=0$ (see [3]), and thus $\mathbb{Q}_{\omega}$ is projective. The following theorem characterizes finitely generated $Z$-torsion free $Z\left[Z_{p}\right]$ modules. The proof is in [2] and in [6].

REINER'S THEOREM. Let $M$ be a finitely generated $Z$-torsion free $Z\left[Z_{p}\right]$ module. Then

$$
M \simeq\left(\bigoplus_{i=1}^{q} \mathbb{Q}_{\omega_{i}}^{(i)}\right) \oplus\left(\bigoplus_{j=1}^{r} \mathscr{B}^{(j)}\right) \oplus Z^{s}
$$

where $\mathbb{Q}^{(i)}$ and $\mathfrak{B}^{(j)}$ are ideals in $C\left(Z\left[\zeta_{p}\right]\right)$, and $Z^{s}$ is free abelian of rank $s$ and trivial as a $Z\left[Z_{p}\right]$ module. Complete isomorphism invariants for $M$ are the ideal class $\mathrm{cl}(M)=\left(\prod_{i=1}^{q} \mathbb{Q}^{(i)}\right) \cdot\left(\prod_{j=1}^{r} \mathscr{B}^{(j)}\right)$ in $C\left(Z\left[\zeta_{p}\right]\right)$, the integers $r$, s, and the rank of $M$ as a $Z$ module.

For $p$ an odd prime, the signed permutation modules are $Z$ and $Z\left[Z_{p}\right]$, and for $p=2, Z, Z\left[Z_{2}\right]$, and $Z_{\varepsilon}=Z\left[\zeta_{2}\right]$. Thus for all primes $p, S$ is the subgroup generated by $[Z]$ and $\left[Z\left[Z_{p}\right]\right]$. In [6] Swan proves that

$$
G_{0}\left(Z\left[Z_{p}\right]\right) \simeq Z \oplus Z \oplus C\left(Z\left[\zeta_{p}\right]\right)
$$

and under this isomorphism

$$
[M]=\left[\left(\bigoplus_{i=1}^{q} \mathbb{Q}_{\omega_{i}}^{(i)}\right) \oplus\left(\bigoplus_{j=1}^{r} \mathscr{B}^{(j)}\right) \oplus Z^{s}\right]
$$

goes to $(q+s, r+q, \mathrm{cl}(M))$. Thus, as in [5], $G_{0}\left(Z\left[Z_{p}\right]\right) / S \simeq C\left(Z\left[\zeta_{p}\right]\right)$, and if $M$ is torsion free, $0=\mathrm{Sw}(M) \Leftrightarrow \mathrm{cl}(M)=1$.

The following lemma will be used in the proof of the main theorem: 
LEMMA (3.1). If $M=\left(\oplus_{i=1}^{q} \mathbb{Q}_{\omega_{i}}^{(i)}\right) \oplus\left(\oplus_{j=1}^{r} \Re^{(j)}\right)$, there is an exact sequence $0 \rightarrow M \rightarrow F \rightarrow F_{1} \oplus Z^{r} \rightarrow 0$ with $F$ and $F_{1}$ free. $F$ and $F_{1}$ may be chosen to be finitely generated if $\mathrm{Sw}(M)=0$.

Proof. Choose $\bar{\omega}_{j} \in \Re^{(j)}, \bar{\omega}_{j} \notin\left(\zeta_{p}-1\right) \mathscr{B}^{(j)}$ and form the exact sequence $0 \rightarrow M \rightarrow P \rightarrow Z^{r} \rightarrow 0$ with $P=\left(\oplus_{i=1}^{q} \mathbb{Q}_{\omega_{j}}^{(i)}\right) \oplus\left(\oplus_{j=1}^{r} \mathscr{B}_{\bar{\omega}}^{(j)}\right)$ by taking the sum of the exact sequences $0 \rightarrow \mathbb{Q}_{\omega_{i}}^{(i)} \rightarrow \mathbb{Q}_{\omega_{i}}^{(i)} \rightarrow 0 \rightarrow 0$ and $0 \rightarrow \mathscr{B}^{(j)} \rightarrow \mathscr{B}_{\bar{\omega} j}^{(j)}$ $\rightarrow Z \rightarrow 0$. Since $P$ is projective, we can choose a free module $F_{1}$ with $F_{1} \oplus P$ $=F$ free. Adding $F_{1}$ to $P$ and to $Z^{r}$ in the sequence yields the exact sequence $0 \rightarrow M \rightarrow F \rightarrow F_{1} \oplus Z^{r} \rightarrow 0 . F_{1}$ can be chosen to be finitely generated if and only if $\mathrm{cl}(M)=\mathrm{cl}(P)$ is trivial in $C\left(Z\left[\zeta_{p}\right]\right)$ by Rim's Theorem (Theorem 6.25 in [3]), and in fact $P$ itself is free in this case. By the remarks preceding the lemma, $\mathrm{Sw}(M)=0 \Leftrightarrow \cdot \dot{\mathrm{cl}}(M)=1$.

We now prove the main theorem:

THEOREM (3.2). Let $A$ be a finitely generated $Z\left[Z_{p}\right]$ module, $Z_{p}$ the cyclic group of prime order $p$. Then there are $Z_{p}$-complexes of type $(A, n) n \geqslant 3$, and there is a finite complex of type $(A, n)$ if and only if $\mathrm{Sw}(A)=0$.

Proof. By the remarks in $\$ 2$, it suffices to prove there are tractable complexes of type $(A, 3)$ where $A$ is torsion free, and finite tractable complexes if $\mathrm{Sw}(A)=0$.

If $A=Z$ with the trivial $Z_{p}$ module structure, we let $S^{1}$ denote the complex numbers of norm one with $Z_{p}$ acting effectively by $t \cdot x=e^{2 \pi i / p} \cdot x . \sum S^{1}$ is then a finite tractable complex of type $(Z, 2)$, where the cellular structure is determined by taking the cone points as 0 -cells, and the line segments through the $p$ pth roots of unity as 1-cells. By suspension we also have finite tractable complexes of type $(Z, n)$ for all $n \geqslant 2$.

In general, let $A=M \oplus Z^{s}$ where $M=\left(\oplus_{i=1}^{q} \mathbb{Q}_{\omega_{i}}^{(i)}\right) \oplus\left(\oplus_{j=1}^{r} \mathscr{B}^{(j)}\right)$ as in Reiner's Theorem. We choose an exact sequence $0 \rightarrow M \rightarrow F \rightarrow F_{1} \oplus Z^{r} \rightarrow 0$ (by Lemma (3.1)), with $F$ and $F_{1}$ free and finitely generated if $\mathrm{Sw}(M)$ $=\mathrm{Sw}(A)=0$. By the above construction and (2.9), there is a tractable complex of type $\left(F_{1} \oplus Z^{r}, 2\right)$ (finite if $\left.\mathrm{Sw}(A)=0\right)$. Therefore by (2.7) there is a tractable complex of type $(M, 3)$ (again finite if $\operatorname{Sw}(A)=0$ ). Finally by (2.9), there is a tractable complex of type $(A, 3)$ (finite if $\mathrm{Sw}(A)=0)$, and by $\S 2$ the proof is complete.

\section{REFERENCES}

1. H. Cartan and S. Eilenberg, Homological algebra, Princeton Univ. Press, Princeton, N. J., 1956. MR 17, 1040.

2. I. Reiner, Integral representations of cyclic groups of prime order, Proc. Amer. Math. Soc. 8 (1957), 142-146. MR 18, 717.

3. D. S. Rim, Modules over finite groups, Ann. of Math. (2) 69 (1959), 700-712. MR 21 \#474. 
4. J.-P. Serre, Cohomologie des groupes discrets, Ann. of Math. Studies, no. 70, Princeton Univ. Press, Princeton, N. J., 1971.

5. R. G. Swan, Invariant rational functions and a problem of Steenrod, Invent. Math. 7 (1969), 148-158. MR 39 \#5532.

6. R. G. Swan and E. G. Evans, K-theory of finite groups and orders, Lecture Notes in Math., vol. 149, Springer, Berlin and New York, 1970. MR 46 \#310.

Department of Mathematics, University of Wisconsin, MiLwaukee, Wisconsin 53201 